\title{
The Qualification Test Design of Reliability Based on EDM
}

\author{
Li Fuping ${ }^{1 ; a}$,Ban Xukeling ${ }^{2 ; b}$, Liu Zhifeng ${ }^{3 ; c}$, Liu Jianyong ${ }^{4 ; d}$ \\ ${ }^{1,2,3}$ Beijing Key Laboratory of Advanced Manufacturing Technology,Beijing University of \\ Technology,Beijing 100124 China \\ ${ }^{4}$ Beijing Institute of Electro Machining,Beijing 100191 China

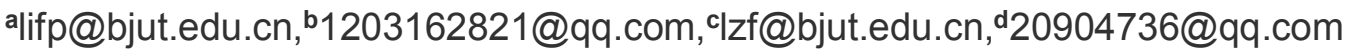

Keywords:electrical discharge machine(EDM);reliability qualification test;truncated sequential test; truncation point

\begin{abstract}
The failure data of electrical discharge machine(EDM)obey weibull distribution. With the mean time between failures(MTBF) as test index, the produce-side risks, user-side risks, identification ratio, MTBF expectation value and shape parameter as known parameter, we design EDM reliability qualification test. For the test requirements that just need to determine the machine reliability and minimize test time and cost,we choose sequential test method. For the shortcoming that sequential test can't end of self when the reliability of the machine tool is at a moderate level,we have to improve and perfect it and we propose the truncated sequential test method. Based on EDM, we compute the receive and reject conditions, censoring time, number of failures of the specific scheme.
\end{abstract}

\section{Introduction}

The reliability level is the key to the quality of electrical discharge machine (EDM) ${ }^{[1]}$. Reliability test is an important method for reliability investigation, reliability evaluation, reliability growth and reliability prediction and distribution ${ }^{[2]}$. Reliability test results can determine whether the product reliability standards, but also for failure analysis, reliability improvement measures to provide a basis for.

The EDM is a typical repairable system and its time between failures obey Weibull distribution ${ }^{[3]}$. So the design of the reliability test program is very complicated, and it is a difficult problem to be solved urgently at present. In the design of EDM reliability qualification test, sometimes only needs to determine the reliability of the machine tool, try to reduce the test time and cost; and sometimes need to obtain more detailed data, provide the basis for improving the reliability of the machine. According to different requirements and conditions, this paper presents two reliability test programs, and combined with the research of large precision six axis EDM designed the reliability test program.

\section{Reliability Test Basis}

Upper and Lower Bounds of Test Index.Reliability test index is usually the mean time between failures MTBF. The upper and lower bounds are expressed separately by $\mathrm{MTBF}_{0}$ and $\mathrm{MTBF}_{1}$.It is generally considered that $\mathrm{MTBF}_{0}$ is the expected value of the manufacturer.

Nominal Value of Decision Risk.Decision risk $\alpha, \beta$ is determined by the production plant and the use of the plant and is the nominal value. The value of decision risk will directly affect fix time censored test scheme design of sample size, test time length and discrimination index -- the size of 
the allowed number of faults. $\langle\text { GJB } 899 \mathrm{~A}-2009\rangle^{[4]}$ pointed out that Risk and use of the manufacturer often take $10 \%, 20 \%$ or $30 \%$.

Identification Ratio.Identification ratio $d$ is the ratio of the Upper and lower bounds of MTBF test. $d=M T B F_{0} / M T B F_{1}$. 《GJB 899A-2009》 [4] pointed out that the most often selected of identification ratio is $1.5,2.0 、 3.0$. The greater the identification ratio, the faster the trial will make the decision, but it will lead to the lowest acceptable MTBF value of the user be reduced, it is necessary to carefully select the appropriate identification ratio.

Shape Parameter.EDM shape parameter $m$ of Weibull distribution in different periods, the shape parameter is different. Literature ${ }^{[5]}$ points out that under the condition of stable production, when the same type of products are not more innovative, the shape parameters are the same. Literature ${ }^{[6]}$ points out that the shape parameter can be replaced by the mean value of the shape parameter estimation of the multi batch of the same type product (or similar product). So the shape parameters of the failure distribution function of EDM in the early fault period can refer to the shape parameters of the same product in the same period.

\section{EDM Truncated Sequential Test}

During the sequential test, continuous or in a short span of time interval of monitoring on the product, according to the cumulative test time and number of failures, each occurrence of a failure, are compared with the pre specified criteria, so as to determine the acceptance, rejection or continue to test ${ }^{[7]}$. When the true value of the product reliability test index is too good or too bad when compared with the expected value of the design plan, the sequential test can be used to judge the reliability of the product quickly.

CNC Machine Sequential Test based on Weibull Model.The probability density function of the two parameter Weibull distribution:

$$
f(t)=\frac{m}{\eta}\left(\frac{t}{\eta}\right)^{m-1} \exp \left[-\left(\frac{t}{\eta}\right)^{m}\right]
$$

Make $q=\frac{m}{\eta^{m}}, \psi=m-1$, mean time between failures(MTBF) can be converted to:

$$
M T B F=\eta \Gamma\left(1+\frac{1}{m}\right)=\left(\frac{\psi+1}{q}\right)^{\frac{1}{\psi+1}} \Gamma\left(1+\frac{1}{\psi+1}\right)
$$

The sequential test design based on the produce-side risks $\alpha$, user-side risks $\beta$,identification ratio $d, \mathrm{MTBF}$ upper bounds $\mathrm{MTBF}_{0}$ and shape parameter $\mathrm{m}$.

First calculate $q_{0}, q_{1}$ of the upper and lower bounds of the MTBF corresponding to:

$$
q_{0}=\frac{m}{\left[\frac{M T B F_{0}}{\Gamma(1+1 / m)}\right]^{m}}, q_{1}=\frac{m}{\left[\frac{M T B F_{1}}{\Gamma(1+1 / m)}\right]^{m}}
$$


In the Weibull distribution $\lambda(t)=\frac{m t^{m-1}}{\eta^{m}}$, so: $m(t)=\frac{q t^{\psi+1}}{\psi+1}$, probability of $\mathrm{R}$ fault in time $\mathrm{t}$ :

$$
P(r)=\left[\frac{q\left(n t^{\psi+1}\right)}{\psi+1}\right]^{r} \exp \left[-\frac{q\left(n t^{\psi+1}\right)}{\psi+1}\right] / r !
$$

The produce-side risks $\alpha$, user-side risks $\beta$, according to the wald ${ }^{[8]}$, $\mathrm{A}$ and $\mathrm{B}$ are set to two constants:

$$
A=\frac{\beta}{1-\alpha}, B=\frac{1-\beta}{\alpha}
$$

Make: $S=\frac{P_{1}(r)}{P_{0}(r)}=\left(\frac{q_{1}}{q_{0}}\right)^{r} \exp \left(-\frac{q_{1}-q_{0}}{\psi+1} n t^{\psi+1}\right)$

So there are the decision condition:

When $S \leq A$, it is considered that the reliability of test product meet the requirements and can be received;

When $S \geq B$, it is considered that the reliability of test product does not meet the requirements and should be rejected;

When $A \leq S \leq B$, it need to continue testing.

The judgment condition of the continuing test is sorted out:

$$
\begin{aligned}
& \ln A<r \ln \frac{q_{1}}{q_{0}}-\frac{q_{1}-q_{0}}{\psi+1} n t^{\psi+1}<\ln B \\
& s r-H_{1}<n t^{\psi+1}<s r+H_{0}
\end{aligned}
$$

Among:

$$
\begin{aligned}
& H_{1}=\frac{-(\psi+1) \ln B}{q_{0}-q_{1}}, H_{0}=\frac{(\psi+1) \ln A}{q_{0}-q_{1}} \\
& S=\frac{-(\psi+1) \ln \left(q_{1} / q_{0}\right)}{q_{0}-q_{1}}
\end{aligned}
$$

Truncated Sequential Test Design.Sequential test can save the total test time and cost, but the product is selective, only for the high or low reliability level product can give full play to this characteristic. Therefore, in order to make up for this shortcoming of sequential test, the method of forced stop test is adopted: To add a truncated for sequential test, make the maximum decision time and maximum fault number of sequential test is limited ${ }^{[9]}$. It is the truncated sequential test.

The key of truncated sequential test is how to determine the truncation point , make: $m=\psi+1, \theta=\frac{m}{q}, t^{m}$ obey the exponential distribution with the parameter of $\theta$, upper and lower bounds of $\theta$ can obtain by $q_{0}, q_{1}$ :

$$
\theta_{0}=\frac{m}{q_{0}} 、 \theta_{1}=\frac{m}{q_{1}}
$$


《GJB 899A-2009》 ${ }^{[4]}$ point out that in order to reduce the difference between practical use and production risks due to the truncated, for predetermined two constants $\mathrm{A}$ and $\mathrm{B}, \mathrm{B}$ need to add a correction factor $\frac{\left(1+d^{\prime}\right)}{2 d^{\prime}}$, so:

$$
A=\frac{\beta}{1-\alpha}, B=\frac{(1-\beta)\left(d^{\prime}+1\right)}{2 \alpha d^{\prime}}
$$

In the formula $d^{\prime}=\frac{\theta_{0}}{\theta_{1}}=\left(\frac{M T B F_{0}}{M T B F_{1}}\right)^{m}=d^{m}$, the $d^{\prime}$ and decision risk $\alpha, \beta$ into the formula, can calculate the value of $A$ and $B$.

After slight modification of the parameters:

$$
s r-H_{1}<n t^{m}<s r+H_{0}
$$

Among:

$$
H_{1}=\frac{-\ln B}{\frac{1}{\theta_{0}}-\frac{1}{\theta_{1}}}, H_{0}=\frac{\ln A}{\frac{1}{\theta_{0}}-\frac{1}{\theta_{1}}}, s=\frac{-\ln \left(\theta_{0} / \theta_{1}\right)}{\frac{1}{\theta_{0}}-\frac{1}{\theta_{1}}}
$$

$n t^{m}$--The total test time of Weibull distribution into exponential distribution

r-- Cumulative number of failures.

According to the formula (9) can draw the image of $\left(n t^{m}-r\right)$ is two parallel oblique-lines, and then draw horizontal line $r=r_{0}$ and vertical line $n t^{m}=T_{0}$. So that the truncated sequential test area can be defined.

For the truncated points $r_{0}$ and $T_{0}$, according to $\langle\text { GJB } 899 \mathrm{~A}-2009\rangle^{[4]}$,first we can select the $\mathrm{r}$ value and the smallest integer $r$ that make the Chi-square distribution formula $\frac{\chi_{\alpha}^{2}(2 r)}{\chi_{1-\beta}^{2}(2 r)} \geq \frac{\theta_{1}}{\theta_{0}}$ set up is $r_{0}$. Then according to $r_{0}$, the truncated test time $T_{0}$ can be calculated by the formula $T_{0}=\frac{\theta_{0} \chi_{\alpha}^{2}\left(2 r_{0}\right)}{2}$.

Truncated sequential test plan of EDM.According to the relevant parameters obtained from EDM manufacturers, the experimental basis is as follows:

The upper bounds of MTBF $M T B F_{0}=600 h$;Nominal value of decision risk $\alpha=\beta=0.2$;Identification ratio $d=2.0$; Shape parameter $m=0.86$.

Specific steps are as follows:

1 ) According to the formula (4) and (8), calculate $\theta_{0} 、 \theta_{1}$ corresponding to the upper and lower bounds of MTBF: $\begin{aligned} & \theta_{0}=229.12 \\ & \theta_{1}=126.23\end{aligned} ;$

2 ) Calculate the correction discrimination ratio $d^{\prime}=d^{m}=1.815$, into the formula (9),get A、B: $A=0.25, B=3.10 ;$ 
3 ) Calculate the parameters in the formula (10): $H_{1}=317.99, H_{0}=389.63, s=167.55$;

4) Calculate the truncation point $r_{0}$ and $T_{0}$. Look up table and calculate: $\theta_{0} / \theta_{1}=0.551$, when degrees of freedom in $2 \mathrm{r}=16$ and $2 \mathrm{r}=18: \frac{\chi_{0.2}^{2}(16)}{\chi_{0.8}^{2}(16)}=0.545, \frac{\chi_{0.2}^{2}(18)}{\chi_{0.8}^{2}(18)}=0.565$. So the smallest integer that makes the formula $\frac{\chi_{\alpha}^{2}(2 r)}{\chi_{1-\beta}^{2}(2 r)} \geq \frac{\theta_{1}}{\theta_{0}}$ set is 9, and then calculate the censoring time: $T_{0}=\frac{\theta_{0} \chi_{\alpha}^{2}\left(2 r_{0}\right)}{2}=1472.90$.

Because there are differences between $\frac{\chi_{0.2}^{2}(18)}{\chi_{0.8}^{2}(18)}=0.565$ and $\theta_{0} / \theta_{1}=0.551$, it is necessary to correct the actual value of decision risk $\alpha^{\prime} 、 \beta^{\prime}$, so make $\frac{\chi_{\alpha}^{2}(18)}{\chi_{1-\beta}^{2}(18)}=0.551$, figure out $\alpha^{\prime}=\beta^{\prime}=0.19$.

From the above calculation, we can draw the truncated sequential test plan:

When $n t^{m} \leq s r-H_{1}=167.55 r-317.99$, it is considered that the reliability of test product meet the requirements and can be received;

When $n t^{m} \geq s r+H_{0}=167.55 r+389.63$, it is considered that the reliability of test product does not meet the requirements and should be rejected;

When $167.55 r-317.99=s r-H_{1}<n t^{m}$, it need to continue testing; $<s r+H_{0}=167.55 r+389.63$

When the number of failure $r_{0}=9$ preferentially reached it is considered that reliability level is not qualified; when the censoring time $T_{0}=14729$ preferentially reached it is considered that reliability level is qualified.

List the truncated sequential test condition table, Table 2-1; draw the condition chart, Figure 2-1:

Table 2-1 the truncated sequential test condition table

\begin{tabular}{ccc}
\hline $\begin{array}{c}\text { the number of } \\
\text { failure }\end{array}$ & total test time $n t^{m}[\mathrm{~h}]$ \\
\hline 0 & - & 389.63 \\
1 & - & 557.18 \\
2 & 17.11 & 724.73 \\
3 & 184.66 & 892.28 \\
4 & 352.21 & 1059.83 \\
5 & 519.76 & 1227.38 \\
6 & 687.31 & 1394.93 \\
7 & 854.86 & 1562.48 \\
8 & 1022.41 & 1730.03 \\
9 & 1189.96 & 1897.58 \\
\hline
\end{tabular}




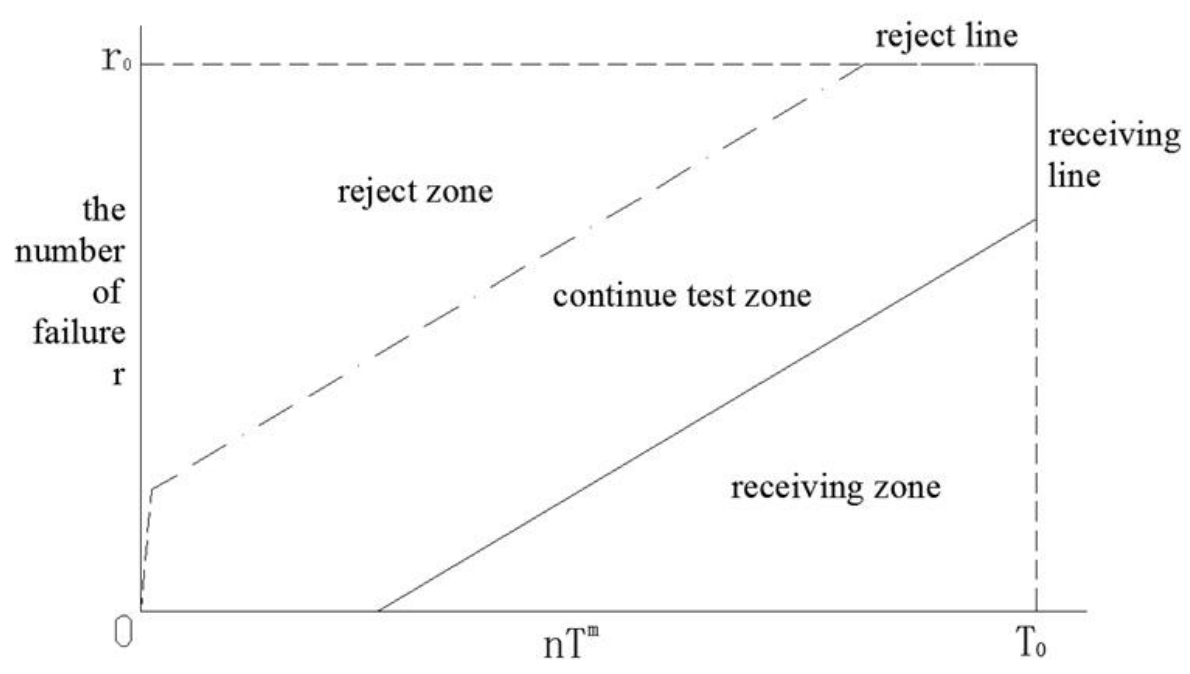

Figure 2-1 the truncated sequential test condition chart

The advantages of truncated sequential test is: When the true value of reliability level is close to the expected value of the reliability level based on experimental design, we can make a decision in time or number of failure censored; And for the high reliability or low product it can be made decision earlier, so as to save the test time and test cost. But also because of this, it will lead to a limited number of data, it is difficult to assess the true value of the reliability level. So the truncated sequential test is very applicable for the test which only need to judge the reliability level is qualified or not.

\section{Conclusion}

Aiming at the EDM fault data obey Weibull distribution, comprehensive consideration of the production risk, the use of risk, the identification ratio, the time limit of the MTBF and the fault distribution shape parameters, we design the reasonable receiving and reject line of sequential test. Further more, we improve the sequential test and get the truncated sequential test plan by adding censoring time and failure number for the sequential test. It provides an efficient test method when manufacturers want to quickly identify the reliability level of EDM and greatly reduce the test time and cost.

\section{Acknowledgements}

Major national science and technology projects,Jing Hua talent project of BJUT (2014ZX04010-011);

Major national science and technology projects,Scale promotion project of high-grade domestic CNC grinding machine reliability(2013ZX04011-013).

\section{Reference}

[1] Jia Yazhou. To improve the reliability of CNC machine is the most important[N].China industry news, 2009-10-26(B01).

[2] Dennis Reidsma, Jean Carletta. Reliability Measurement without Limits[J].Computational Linguistics,2008,34(3):319-326.

[3] Wu Junqi. The reliability time model and maintainability index evaluation of CNC machine tool[D].Changchun: Jilin University,2005.

[4] General Armament Department of the people's Liberation Army Standardization research center of electronic information base. GJB 899A-2009 Reliability evaluation and acceptance test[S]// 
Approved by the General Armament Department of the Chinese people's Liberation Army.Beijing: China Standard Press,2009.

[5] Li Zuodong. The reliability test and evaluation of Weibull distribution[J].Quality and reliability,2003(3):26-27.

[6] Zhou Yuanquan, Shen Fengxian. Reliability acceptance plan of Repairable System[J].Quality and reliability,2011(151):10-12.

[7] Michael G.Pecht. Reliability Engineering Foundation [M].Electronic Industry Publishing House,2011.5.

[8] Dai Shusen, etc. Reliability test and statistical analysis (2)[M].Beijing:National Defence Industry Press, 1983.1.

[9] Chen Bingkun. Research on reliability test design and evaluation method of CNC machine tool [D].Doctoral Dissertation of Jilin University,2011.12. 